University of Nebraska - Lincoln

DigitalCommons@University of Nebraska - Lincoln

Uniformed Services University of the Health

Sciences

U.S. Department of Defense

2011

\title{
EPR measurements of fingernails in Q-band
}

\author{
Alex Romanyukha \\ Naval Dosimetry Center, Alexander.Romanyukha@med.navy.mil \\ François Trompier \\ Institut de Radioprotection et de Sûreté Nucléaire \\ Ricardo A. Reyes \\ Uniformed Services University of the Health Sciences \\ Mark A. Melanson \\ Armed Forces Radiobiology Research Institute
}

Follow this and additional works at: https://digitalcommons.unl.edu/usuhs

Part of the Medicine and Health Sciences Commons

Romanyukha, Alex; Trompier, François; Reyes, Ricardo A.; and Melanson, Mark A., "EPR measurements of fingernails in Q-band" (2011). Uniformed Services University of the Health Sciences. 86.

https://digitalcommons.unl.edu/usuhs/86

This Article is brought to you for free and open access by the U.S. Department of Defense at DigitalCommons@University of Nebraska - Lincoln. It has been accepted for inclusion in Uniformed Services University of the Health Sciences by an authorized administrator of DigitalCommons@University of Nebraska Lincoln. 


\title{
EPR measurements of fingernails in Q-band
}

\author{
Alex Romanyukha ${ }^{\mathrm{a}, *}$, François Trompier ${ }^{\mathrm{b}}$, Ricardo A. Reyes ${ }^{\mathrm{c}}$, Mark A. Melanson ${ }^{\mathrm{d}}$ \\ ${ }^{a}$ Naval Dosimetry Center, Bethesda, MD, USA \\ ${ }^{\mathrm{b}}$ Institut de Radioprotection et de Sûreté Nucléaire, Fontenay-aux-Roses Cedex, France \\ ${ }^{\mathrm{c}}$ Uniformed Services University of the Health Sciences, Bethesda, MD, USA \\ ${ }^{\mathrm{d}}$ Armed Forces Radiobiology Research Institute, Bethesda, MD, USA
}

\section{A R T I C L E I N F O}

\section{Article history:}

Received 22 November 2010

Received in revised form

10 February 2011

Accepted 1 April 2011

\section{Keywords:}

EPR dosimetry

Fingernails

Q-band

Accidental dosimetry

Radiation dosimetry

\begin{abstract}
A B S T R A C T
Results of a feasibility study for the use of the Q-band EPR measurements of fingernails are presented. Details of the first protocol developed for Q-band $(34 \mathrm{GHz})$ EPR dose measurements in fingernails and preliminary results of a dosimetry study in comparison with the commonly-used X-band $(9 \mathrm{GHz})$ are reported. It was found that $1-5 \mathrm{mg}$ sample mass was sufficient for EPR measurements in fingernails in the Q-band, which is significantly less than the 15-30 mg needed for the X-band. This finding makes it possible to obtain sufficient fingernail sample for dose measurements, practically from every finger of any person. Another finding was that the spectral resolution of the mechanically-induced signal (MIS) and radiation-induced signal (RIS) in the Q-band was significantly better than in the X-band. The RIS and MIS in the Q-band spectrum have a more complex structure than in the X-band, which potentially offers the possibility to do dose measurements in fingernails without treatment and immediately after clipping. These findings and recent results related to fingernail dosimetry in the Q-band and its perspectives are discussed here.
\end{abstract}

Published by Elsevier Ltd.

\section{Introduction}

Significant progress in the development of electron paramagnetic resonance (EPR) dosimetry in fingernails has been achieved in recent years (see Reyes et al., 2008, 2009; Romanyukha et al., 2007b, 2010; Trompier et al., 2007, 2009). Chemically, fingernails are mainly composed of alpha keratin, a protein made of three alpha helical peptide chains, twisted in a left-handed coil that is strengthened by disulfide bridges from adjacent cystein groups. It has been demonstrated that EPR dosimetry with fingernails has a relatively low dose limit (1-2 Gy) and simple sampling processing, which allows the possibility of completing radiation dose assessments in a short time of about $20 \mathrm{~min}$. Such features make EPR fingernail dosimetry an attractive methodology for dose evaluations during mass casualty events involving radioactive sources. It has also been well established that a component of the radiation-induced EPR signal (RIS) in fingernails is persistent and proportional to the radiation dose. If necessary, this signal can be preserved for long time (months) by storage at low temperatures (on ice or in freezer). However, there are some known problems with the practical application of EPR fingernail dosimetry. From our point of view, three issues are the most critical to resolve:

\footnotetext{
* Corresponding author. Tel.: +1 301295 4149; fax: +1 3012954165 .

E-mail address: Alexander.Romanyukha@med.navy.mil (A. Romanyukha).
}

1. The presence of so-called mechanically-induced signals (MIS1 and MIS2) in the EPR spectrum of fingernails. These MISs obscure the RIS at low doses ( $<5 \mathrm{~Gy}$ ). Water treatment of fingernails prior to dose measurements was first suggested by Trompier et al. (2007) to reduce the MIS intensity. However, a persistent component is observed with similar spectral characteristics as RIS, which can induce an error in dose estimation.

2. Variability of radiation sensitivity and RIS shape. According to Reyes et al. (2009), the major factor responsible for variation of radiation sensitivity in fingernails is its water content, which can affect radiation sensitivity of fingernails up to $35 \%$. The major factor responsible for the type (linear or exponential decay) of dose dependence of radiation-induced signal intensity was identified as the mechanical stress in fingernails at their cutting.

3. Need to have sufficient amount of sample for EPR dose measurements (at least $15 \mathrm{mg}$ ), which is not always feasible. Furthermore, it is possible that fingernails from different fingers may receive quite different radiation doses as result of an accident. Therefore, a combination of fingernail clippings from different fingers in a single sample will not always provide an accurate dose assessment.

In an attempt to overcome the problems mentioned above, we tested the use of the Q-band $(34 \mathrm{GHz})$ for EPR measurements in fingernails instead of the commonly-used X-band (9 GHz). The 
Table 1

List of the used parameters of the EPR measurements of fingernail samples in Q-band.

\begin{tabular}{ll}
\hline Parameter & Value \\
\hline Mw frequency & $34.2 \mathrm{GHz}$ \\
Resonator model & ER 5106QT \\
Sample mass & $1.5-3.0 \mathrm{mg}$ \\
Inner diameter of sample tube & $2 \mathrm{~mm}$ \\
Incident mw power & $1 \mathrm{~mW}$ \\
Sweep width & $16 \mathrm{mT}$ \\
HF modulation & $100 \mathrm{kHz}$ \\
Amplitude of HF modulation & $0.45 \mathrm{mT}$ \\
Receiver gain & $60 \mathrm{db}$ \\
Time constant & $41 \mathrm{~ms}$ \\
Number of points & 1024 \\
Sweep time & $41.96 \mathrm{~s}$ \\
Number of scans & 20 \\
Total recording time & $15 \mathrm{~min}$ \\
\hline
\end{tabular}

Q-band is known to have better spectral resolution and higher sensitivity than the X-band. However, it is much more vulnerable to the high water content of samples under measurement. Therefore, the choice of an optimal microwave band for EPR measurements is not trivial and requires comparative studies. In case of tooth enamel, such a comparative study was done by Romanyukha et al. (2007a); however, there is no such published report for fingernails. The goals of this paper are following:

- Develop a protocol for EPR measurements of fingernails in Q-band;

- Compare appearance of the known effects (mechanical stress, water treatment, radiation, microwave power dependence) in Q- and X-bands;

- Evaluate possible benefits of measurements in Q-band, mainly investigating how the improved spectral resolution
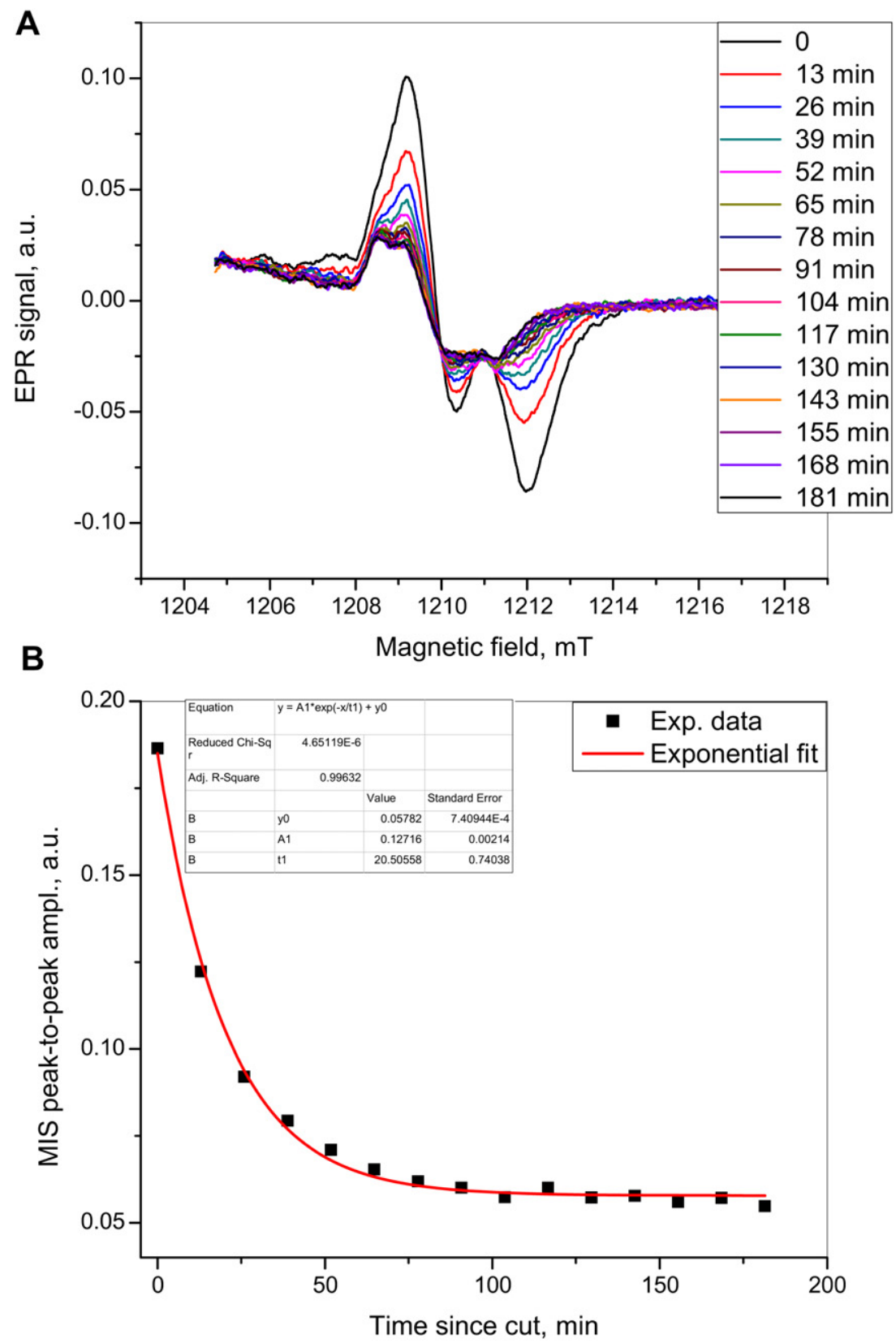

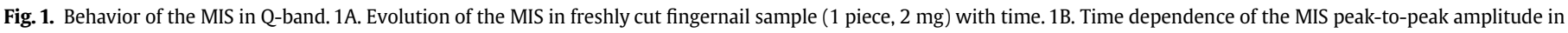
the same sample. Solid line shows exponential decay fit of experimental data. 
allows the use of untreated samples for dose measurements immediately after cutting and without treatment with water.

\section{Materials and methods}

Fifteen samples in total, collected from four different donors, were used in this work. All studied fingernails were collected from donors the same day of EPR measurements. All dose dependences were obtained during one day by sequential irradiations of the sample under study. Sharp scissors were used to cut the fingernails and all pieces of fingernails were approximately $0.5-1.5 \mathrm{~mm}$ wide and $2-3 \mathrm{~mm}$ long. The typical sample mass was $1-6 \mathrm{mg}$. The water used during rinsing steps and for solutions had $18.2 \mathrm{M}$ purity (deionized water). Calibrated ${ }^{137} \mathrm{Cs}$ and ${ }^{60} \mathrm{Co}$ radiation sources were used for irradiation of the fingernail samples. Because of their different dose rates, the ${ }^{137} \mathrm{Cs}$ source was used for irradiation to relatively low doses ( $<10 \mathrm{~Gy}$ ); whereas, the ${ }^{60}$ Co source was used for irradiation to higher doses ( $>10 \mathrm{~Gy}$ ). EPR measurements were carried out on a Q-band EPR spectrometer, Bruker EMXplus (Bruker BioSpin). Spectrometer parameters, used to record EPR spectra of fingernails in this study, are listed in the Table 1 . There was no intensity standard used in the EPR measurements. The Q-band allows only a relatively small amount of sample to be placed into the resonator and the mw-bridge tuning cannot always (depending on sample size and its water content) tolerate the simultaneous presence of standard and test samples inside. In order to make possible relative quantitative measurements in Q-band (for example, dose dependence of EPR dose response) a special technique of resonator tuning was developed. This technique is different from one used in the X-band and allows to keep the same resonator tuning parameters (resonator length, mw frequency, and mw-phase) for different samples. The developed tuning technique was tested by comparison of EPR dose responses from different fingernail and tooth enamel samples irradiated to the same dose. The different samples irradiated to the same dose were verified to have the same or close mass-normalized dose response.

\section{Results and discussion}

Fig. 1 shows the fading evolution of the MIS EPR spectra with time in freshly-cut unirradiated fingernail sample (1 piece, $2 \mathrm{mg}$ ) measured in Q-band. When comparing the MIS appearance in the $\mathrm{X}$-band to that in the Q-band, no significant differences are

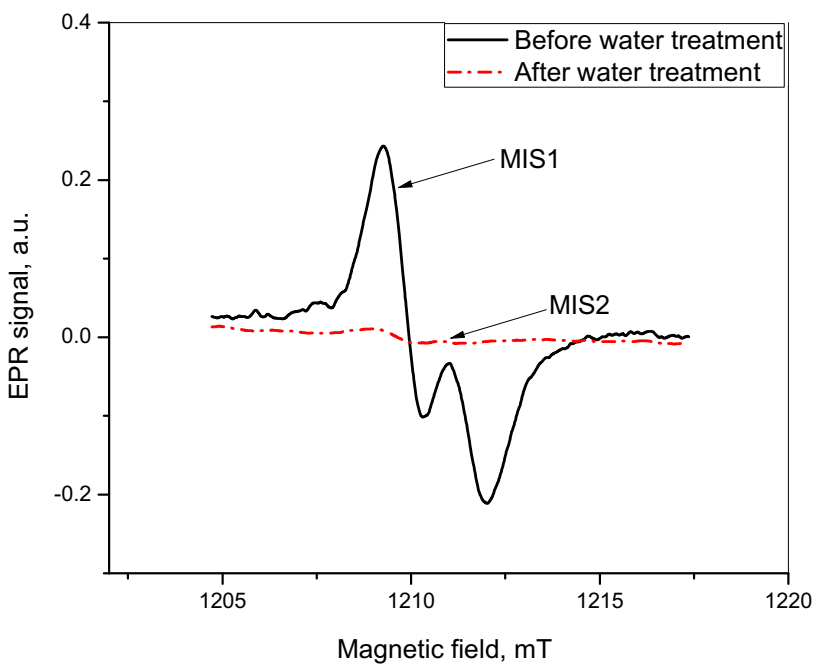

Fig. 2. Effect of water treatment in unirradiated fingernail sample (1 piece, $2 \mathrm{mg}$ ). observed in the spectra recorded immediately after the cuts of the nails (Chandra and Symons, 1987; Romanyukha et al., 2007b; Reyes et al., 2008, 2009; Wilcox et al., 2010; Black and Swarts, 2010). Although Q-band does not allow distinguishing more than the two main components of the MIS already observed in X-band: MIS1 (the doublet) and MIS2 (the singlet) (Reyes et al., 2009; Wilcox et al., 2010; Black and Swarts, 2010); the EPR spectrum measured at $181 \mathrm{~min}$ in Fig. 1 demonstrates some improvement in spectral resolution in Q-band, the doublet structure is obviously observed without any mathematical treatment of the spectra, whereas such structure is not seen in X-band after a similar time delay (compare our Figs. 1 and 2 in Wilcox et al., 2010). One can see some resembles in the shape of EPR spectrum measured at $181 \mathrm{~min}$ and MIS spectrum depicted on Fig. 3 in Wilcox et al. (2010). Moreover in case of Q-band it does not require spectra subtraction to see a structure, which was once called in Wilcox et al. (2010) as a transient signal MIS3. After about one day residual MIS signal stops to decay and its shape becomes a singlet, which was what named in Reyes et al. (2008) as MIS2.

In order to reduce the MIS intensity, a procedure of short-time (5-10 min) soaking in water was suggested by Trompier et al. (2007) and further developed in Reyes et al., 2008, 2009. Fig. 2 demonstrates the effect of this procedure as measured in the Q-band. One can see that this procedure works well in the Q-band
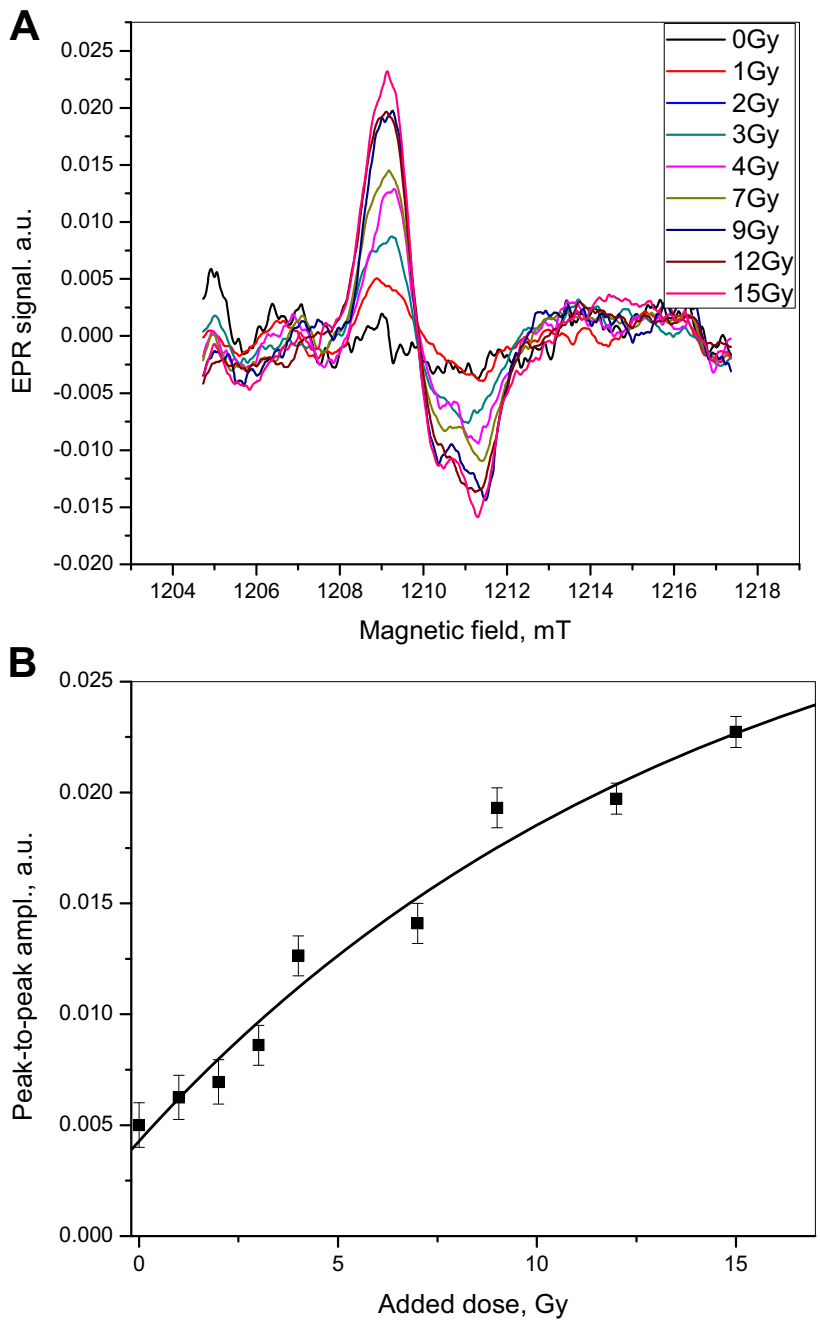

Fig. 3. (A) RIS change with increase of dose in water-treated sample (1 piece, $5 \mathrm{mg}$ ). (B) Dose dependence of RIS peak-to-peak amplitude in water-treated sample ( 1 piece, $5 \mathrm{mg}$ ). Solid line shows exponential decay fit of experimental data. 
using a very small sample ( $2 \mathrm{mg}$ ). As it was discussed by Reyes et al. (2008) soaking in water effectively eliminate MIS1, while MIS2 (stable mechanically-induced component) has a trend of slow increase with time after treatment. For convenience MIS1 and MIS2 are shown on Fig. 2. As compared with spectra appearance in Wilcox et al. (2010) a remarkably better signal-to-noise ratio in the Q-band, as compared to the X-band can also be observed.

Fig. $3 \mathrm{~A}$ and $\mathrm{B}$ show the RIS change with dose and corresponding dose dependence in water-treated sample prior irradiation; whereas, Fig. 4A and B show the same dependences in untreated fingernail samples. As it was previously discussed in detail by Reyes et al. (2009), the RIS behavior in these two types of samples are very different: untreated fingernails (mechanically-stressed state) demonstrate linear dose dependence; while treated fingernails show non-linear dose dependence with saturation starting at about 15-20 Gy. Radiation sensitivity of untreated samples was also found to be significantly higher than water-treated samples but the RIS in untreated samples is significantly less stable. Until now, there is no full understanding of such remarkable difference in the behavior of the RIS in water-treated and untreated samples, since in $\mathrm{X}$-band RIS in untreated and water treated were found to be similar. The EPR measurements in Q-band offer a better understanding of this phenomenon. Fig. 5 shows a qualitative (non-scaled) comparison of the RIS in irradiated water-treated and untreated fingernail
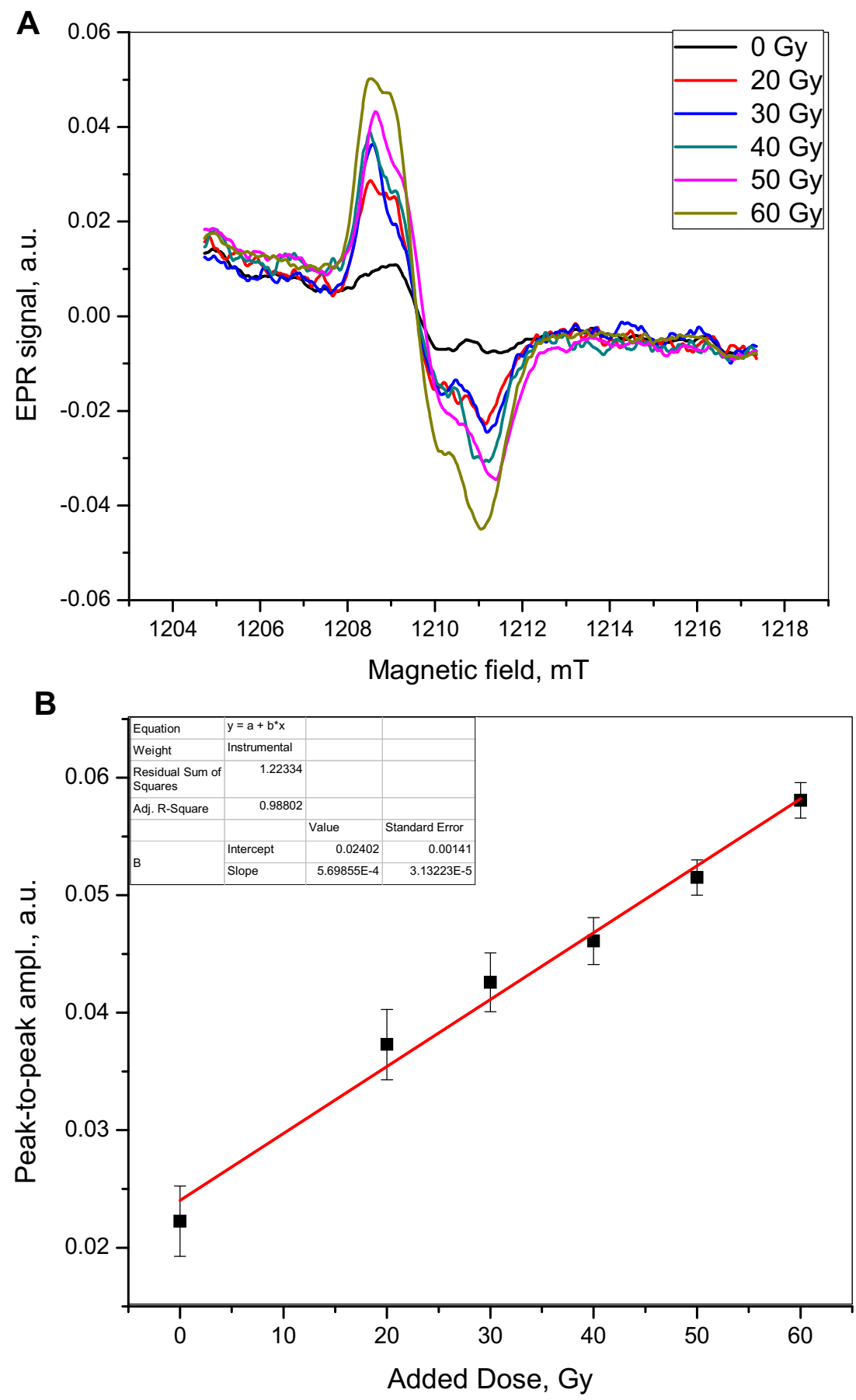

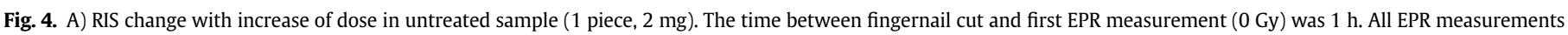

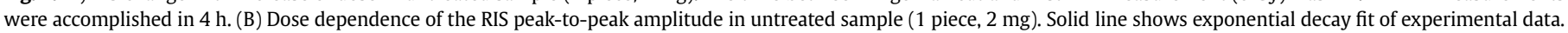




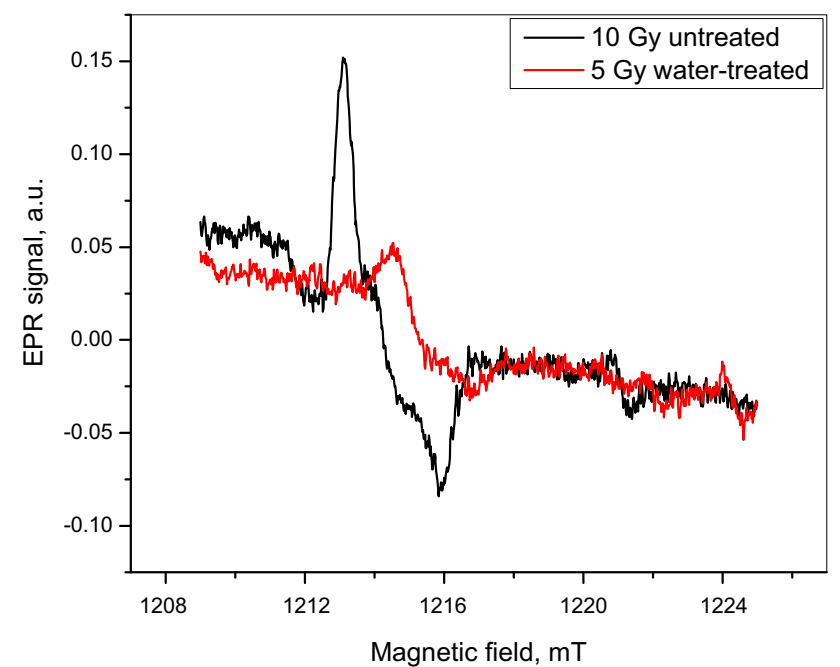

Fig. 5. Qualitative comparison of the effect of radiation in treated and untreated freshly-cut samples. There is only one RIS component in the treated sample. This shows the difference between the RIS in treated and untreated samples.

samples. One can see from this figure that the RIS in untreated fingernails has a complex structure; whereas, in water-treated samples it is singlet. The RIS in untreated samples using the Q-band has a resolved structure, which allows identifying similarities and differences with the RIS in treated samples. It appears from Fig. 5 that there are two types of radiation-induced radicals in untreated samples. One type of radiation-induced signals is more intense (narrow intense component on left part of spectrum of the untreated fingernail sample), and does not show saturation until high doses ( $\sim 100 \mathrm{~Gy}$ ) but is significantly less stable with time. The second component of the RIS in the untreated sample (located in the central part of the spectrum on Fig. 5) looks similar to the RIS in water-treated sample. This part of the RIS is significantly less intense but it is very stable. The opportunity to resolve the RIS in untreated fingernail samples suggests a way to measure the more stable component of the RIS in these samples without water treatment. Further studies should be conducted on a bigger number of fingernail samples collected from different donors. This can augment the significance of our results and considerably simplify and accelerate radiation dose measurements in fingernails.

\section{Conclusions}

This feasibility study of fingernails using the Q-band showed that this microwave band works significantly better for fingernails than the commonly used X-band, providing better signal-to-noise ratio in very small samples ( $\sim 2 \mathrm{mg}$ ). Developed procedure of Q-band microwave bridge tuning allows to produce reliable EPR dose measurements in small samples of fingernails. Furthermore, most features of fingernails observed in the X-band, e.g. the same spectral components (MIS1, MIS2 and RIS), were also found in the Q-band but with more details. The most important result of this study is an observation of the RIS complex structure in untreated fingernail samples, e.g. presence of two components. One of the RIS components in untreated samples is similar to that found in water-treated samples (less intense and stable). Whereas, its other component is more intense, less stable, and is not observed in water-treated samples. This gives an opportunity to develop a procedure to measure RIS in freshly-cut fingernails without a delay and/or application of a water treatment. Certainly, more measurements in samples collected from various fingernail donors need to be done before finalizing a protocol of the dose measurements in fingernails using the Q-band.

\section{Disclaimer}

The study was funded through U.S. Department of Defense operational and maintenance budget and IRSN budget. The funding sources did not play any role in this study design, the collection, analysis and interpretation of data. The views expressed in this paper are those of the authors and do not reflect the official policy or position of the Navy and Marine Corps Public Health Center, Navy Bureau of Medicine and Surgery, Department of the Navy, Department of Defense, or the U.S. Government.

\section{References}

Black, P.J., Swarts, S.G., 2010. Ex vivo analysis of irradiated fingernails: chemical yields and properties of radiation-induced and mechanically-induced radicals. Health Phys. 98, 301-308.

Chandra, H., Symons, M.C.R., 1987. Sulphur radicals formed by cutting alpha-keratin. Nature 328, 833-847.

Reyes, R.A., Romanyukha, A., Trompier, F., Mitchell, C.A., Clairand, I., De, T., Benevides, L.A., Swartz, H.M., 2008. Electron paramagnetic resonance in human fingernails: the sponge model implication. Radiat. Environ. Biophys. 47, 515-526.

Reyes, R.A., Romanyukha, A., Olsen, C., Trompier, F., Benevides, L.A., 2009. Electron paramagnetic resonance in irradiated fingernails: variability of dose dependence and possibilities of initial dose assessment. Radiat. Environ. Biophys. 48, 295-310.

Romanyukha, A., Mitchell, C.A., Schauer, D.A., Romanyukha, L., Swartz, H.M., 2007a. Q-band EPR biodosimetry in tooth enamel microprobes: feasibility test and comparison with X band. Health Phys. 93, 631-635.

Romanyukha, A., Trompier, F., LeBlanc, B., Calas, C., Clairand, I., Mitchell, C.A. Smirniotopoulos, J.G., Swartz, H., 2007b. EPR dosimetry in chemically treated fingernails. Radiat. Meas. 42, 1110-1113.

Romanyukha, A., Reyes, R.A., Trompier, F., Benevides, L.A., 2010. Fingernail dosimetry: current status and perspectives. Health Phys. 98 (2), 296-300.

Trompier, F., Kornak, L., Calas, C., Romanyukha, A., LeBlanc, B., Clairand, I., Mitchell, C.A., Swartz, H., 2007. Protocol for emergency EPR dosimetry in fingernails. Radiat. Meas. 42, 1085-1088.

Trompier, F., Romanyukha, A., Kornak, L., Calas, C., LeBlanc, B., Mitchell, C., Swartz, H., Clairand, I., 2009. Electron paramagnetic resonance radiation dosimetry in fingernails. Radiat. Meas. 44, 6-10.

Wilcox, D.E., He, X., Gui, J., Ruuge, A.E., Li, H., Williams, B.B., Swartz, H.M., 2010 Dosimetry based on EPR spectral analysis of fingernail clippings. Health Phys. 98, 309-317. 